\title{
The impact of Aboriginal status, cigarette smoking and smoking cessation on perinatal outcomes in South Australia
}

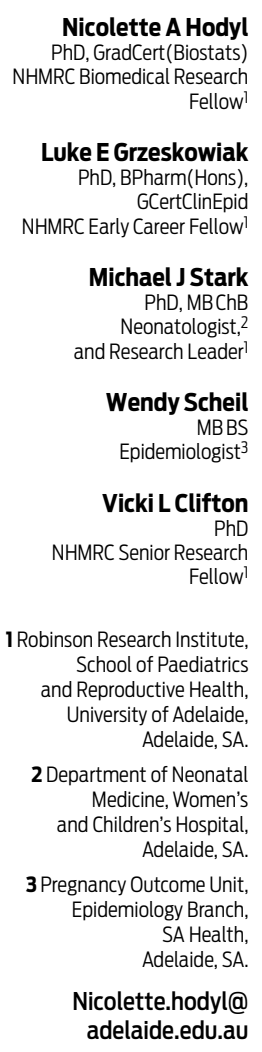

MJA 2014; 201: 274-278 doi: $10.5694 /$ mjal3.11142
T here are clear disparities in health outcomes between the Aboriginal and non-Aboriginal populations, exemplified in the high rates of adverse perinatal outcomes. For example, much higher rates of stillbirth (13.3 versus 7.1 per 1000 live births), neonatal deaths (6.9 versus 2.7 per 1000 live births) and preterm deliveries (13.7\% versus $7.9 \%)^{1-3}$ are observed in Aboriginal relative to non-Aboriginal Australians. Further, Aboriginal babies have an average birthweight about $200 \mathrm{~g}$ lighter than non-Aboriginal babies, and more than double the incidence of low birthweight (12.5\% versus $5.9 \%$ Australia-wide). ${ }^{3}$

Smoking during pregnancy is a well established risk factor for poor perinatal outcomes. ${ }^{4}$ A Cochrane review showed that smoking interventions in pregnancy reduce the relative risk of low birthweight by $17 \%$ and preterm birth by $14 \%$, and increase mean birthweight by 54 g. ${ }^{5}$ While cigarette smoking rates have decreased in Australia over the past few decades, rates of smoking among Aboriginal women are three times higher than those among non-Aboriginal women. ${ }^{2}$ Given these statistics, a reduction in maternal smoking during pregnancy is potentially the single most effective short-term method of improving perinatal outcomes for Aboriginal women. ${ }^{1}$ The success of smoking cessation during pregnancy in these women, however, appears to be affected by socioeconomic disadvantage, low education levels and stressful life circumstances. ${ }^{6-9}$ The introduction of culturally sensitive programs in single sites around Australia has had some benefit. ${ }^{10-14}$ A concerted national approach to sustain such changes requires recognition of the impact of smoking reduction and cessation during pregnancy in improving perinatal outcomes. In our study, therefore, we aimed to identify the contribution of active cigarette smoking and

Abstract

Objective: To assess the impact of Aboriginal status, active cigarette smoking and smoking cessation during pregnancy on perinatal outcomes.

Design: Retrospective cohort study from 1 January 1999 to 31 December 2008.

Setting: All singleton births in South Australia.

Participants: Population-based birth records of pregnancies to Aboriginal women $(n=4245)$ and non-Aboriginal women $(n=167746)$.

Main outcome measures: Adjusted odds ratios (aORs) and 95\% Cls for adverse maternal and neonatal outcomes according to Aboriginal status and maternal smoking in pregnancy.

Results: Active cigarette smoking during pregnancy was associated with an increased risk of adverse perinatal outcomes, including premature labour (Aboriginal, 1-10 cigarettes per day: aOR, 1.69; 95\% Cl, 1.28-2.23; non-Aboriginal, 1-10 cigarettes per day: aOR, 1.46; 95\% Cl, 1.34-1.58), preterm birth (Aboriginal, 1-10 cigarettes per day: aOR, 1.40; 95\% Cl, 1.14-1.73; non-Aboriginal, 1-10 cigarettes per day: aOR, 1.48; $95 \% \mathrm{Cl}, 1.39-1.57$ ), intrauterine growth restriction (Aboriginal, 1-10 cigarettes per day: aOR, 2.33; 95\% Cl, 1.77-3.08; non-Aboriginal, 1-10 cigarettes per day: aOR, 2.65; 95\% Cl, 2.48-2.83) and small for gestational age (Aboriginal, 1-10 cigarettes per day: aOR, 2.49; 95\% Cl, 2.06-3.00; nonAboriginal, 1-10 cigarettes per day: aOR, 2.29; $95 \%$ Cl, 2.20-2.40). For both Aboriginal and non-Aboriginal women who smoked 11 or more cigarettes per day the aOR for these outcomes increased. Smoking cessation in the first trimester reduced these risks to levels comparable with non-smokers. The risk of each adverse outcome was greater in Aboriginal than non-Aboriginal women for all smoking categories; however, interactions between Aboriginal status and smoking were not significant, indicating an equal contribution of smoking to poor outcomes in both populations.

Conclusions: Smoking cessation or reduction during pregnancy would significantly improve outcomes in both Aboriginal and non-Aboriginal women. This should be made a clear priority to improve pregnancy outcomes for all women.

smoking cessation on maternal and neonatal pregnancy outcomes in a large cohort of Australian Aboriginal and non-Aboriginal women in South Australia.

\section{Methods}

The perinatal outcomes were derived from a database of mandatory information collected by the SA Department of Health. The information in this collection has been validated and proven reliable. ${ }^{15}$ Information was extracted for all singleton births from 1 January 1999 to 31 December 2008. Women were identified as Aboriginal if they identified themselves as either Australian Aboriginal and/or Torres Strait Islander (herein referred to as Aboriginal). Multiple births were excluded due to the increased independent risk of adverse outcomes in these pregnancies. The study was approved by the SA Department of Health Human Research Ethics Committee, the South Australian Aboriginal Health Research and Ethics Committee, and the University of Adelaide Human Research Ethics Committee.

Women's cigarette smoking status was collected at the first antenatal visit, and the number of cigarettes smoked per day was recorded after 20 weeks' gestation. These data were used to classify women as either nonsmokers, those who quit during pregnancy or smokers, according to the average quantity of cigarettes smoked each day in the second half of pregnancy (either 1-10 or 11+ cigarettes per day). The Australian Bureau of Statistics Socio-Economic Indexes for Areas (SEIFA) population-based 
1 Adjusted odds ratios $(95 \% \mathrm{Cl}$ ) for perinatal outcomes in Aboriginal women according to smoking in pregnancy, using non-Aboriginal women with matched smoking status as the reference group

\begin{tabular}{|c|c|c|c|c|c|}
\hline Perinatal outcomes & $\begin{array}{l}\text { Aboriginal, } \\
\text { non-smoker }\end{array}$ & $\begin{array}{l}\text { Aboriginal, quit } \\
\text { smoking }\end{array}$ & $\begin{array}{l}\text { Aboriginal, smokes 1-10 } \\
\text { cigarettes per day }\end{array}$ & $\begin{array}{l}\text { Aboriginal, smokes } 11+ \\
\text { cigarettes per day }\end{array}$ & $\begin{array}{c}P \text { overall interaction } \\
\text { (Aboriginal status } \times \text { smoking) }\end{array}$ \\
\hline $\begin{array}{l}\text { Hypertensive disorders of } \\
\text { pregnancy* }\end{array}$ & $1.40(1.19-1.64)^{\dagger}$ & $1.28(0.83-1.98)$ & $1.11(0.88-1.41)$ & $1.38(1.03-1.86)^{\ddagger}$ & 0.08 \\
\hline Gestational diabetes* & $2.72(2.26-3.27)^{\dagger}$ & $1.17(0.58-2.35)$ & $1.75(1.32-2.34)^{\dagger}$ & $1.74(1.27-2.38)^{\dagger}$ & $<0.001$ \\
\hline Antepartum haemorrhage* & $1.08(0.83-1.41)$ & $0.86(0.42-1.78)$ & $0.86(0.66-1.12)$ & $0.89(0.64-1.24)$ & 0.74 \\
\hline Urinary tract infection* & $1.81(1.46-2.22)^{\dagger}$ & $2.53(1.55-4.11)^{\dagger}$ & $1.95(1.58-2.41)^{\dagger}$ & $1.72(1.30-2.28)^{\dagger}$ & 0.56 \\
\hline Elective caesarean* & $1.23(1.06-1.43)^{5}$ & $0.89(0.56-1.41)$ & $1.10(0.91-1.32)$ & $0.94(0.74-1.19)$ & 0.46 \\
\hline Emergency caesarean* & $1.73(1.51-1.98)^{\dagger}$ & $1.53(1.08-2.17)^{\ddagger}$ & $1.28(1.09-2.50)^{5}$ & $1.43(1.17-1.76)^{\dagger}$ & 0.11 \\
\hline Premature labour* & $1.82(1.46-1.27)^{\dagger}$ & $2.01(1.17-3.46)^{\ddagger}$ & $1.77(1.45-2.15)^{\dagger}$ & $1.79(1.41-2.29)^{\dagger}$ & 0.47 \\
\hline PROM* & $1.93(1.46-2.55)^{\dagger}$ & $2.26(1.11-4.61)^{\ddagger}$ & $1.71(1.31-2.25)^{\dagger}$ & $2.38(1.77-3.21)^{\dagger}$ & 0.77 \\
\hline Very preterm birth $<32$ weeks* & $2.70(2.07-3.51)^{\dagger}$ & $3.31(1.64-6.65)^{\dagger}$ & $1.79(1.36-2.35)^{\dagger}$ & $2.06(1.48-2.88)^{\dagger}$ & 0.42 \\
\hline Preterm birth < 37 weeks* & $2.12(1.81-2.47)^{\dagger}$ & $2.56(1.71-3.83)^{\dagger}$ & $1.71(1.46-2.00)^{\dagger}$ & $1.75(1.44-2.12)^{\dagger}$ & 0.42 \\
\hline IUGR (<3rd percentile) & $1.66(1.32-2.08)^{\dagger}$ & $2.41(1.38-4.18)^{\dagger}$ & $1.49(1.25-1.78)^{\dagger}$ & $1.55(1.25-1.92)^{\dagger}$ & 0.59 \\
\hline SGA $(<10 \text { th percentile })^{\natural}$ & $1.44(1.24-1.67)^{\dagger}$ & $1.74(1.20-2.53)^{5}$ & $1.56(1.38-1.77)^{\dagger}$ & $1.32(1.12-1.54)^{\dagger}$ & 0.19 \\
\hline Neonatal intensive care** & $1.86(1.45-2.39)^{\dagger}$ & $2.43(1.22-4.84)^{\ddagger}$ & $1.55(1.17-2.04)^{\S}$ & $1.61(1.14-2.27)^{5}$ & 0.64 \\
\hline Neonatal resuscitation** & $1.29(1.11-1.50)^{\dagger}$ & $1.16(0.76-1.78)$ & $1.12(0.94-1.34)$ & $0.98(0.78-1.24)$ & 0.23 \\
\hline Oxygen therapy (> 4 hours) ${ }^{* *}$ & $1.67(0.40-1.99)^{\dagger}$ & $1.66(1.01-2.74)^{\ddagger}$ & $1.45(1.19-1.77)^{\dagger}$ & $1.35(1.05-1.75)^{\ddagger}$ & 0.56 \\
\hline Stillbirth* & $2.82(1.88-4.25)^{\dagger}$ & $1.61(0.37-6.97)$ & $1.73(1.06-2.81)^{\ddagger}$ & $1.80(0.96-3.39)$ & 0.42 \\
\hline Neonatal death $(<28 \text { days })^{\dagger \dagger}$ & $2.23(1.08-4.59)^{\ddagger}$ & 3.55 (0.44-28.99) & $2.22(1.01-4.87)^{\ddagger}$ & $2.19(0.82-5.88)$ & 0.96 \\
\hline Congenital abnormalities* & $1.16(0.86-1.57)$ & $1.21(0.52-2.81)$ & $1.10(0.79-1.54)$ & $1.47(0.99-2.19)$ & 0.78 \\
\hline
\end{tabular}

quintile scores, derived from home address postcode, were used to indicate socioeconomic status. ${ }^{16}$ Small for gestational age (SGA) was defined as less than the 10th birthweight percentile. Intrauterine growth restriction (IUGR) was defined as less than the third customised birthweight percentile. Other maternal demographic, obstetric and neonatal conditions have been defined elsewhere. ${ }^{17}$

Pregnancy outcomes were analysed with univariate and multivariate regression modelling to assess the impact of Aboriginal status and smoking (and the interaction between smoking and Aboriginal status) on perinatal outcomes. These models were adjusted for potential confounders identified a priori, and reported as odds ratios (ORs) with 95\% confidence intervals. Post-hoc comparisons evaluated the impact of pregnancy smoking status among Aboriginal and non-Aboriginal women separately, using three groups in comparison with a control (reference) group. The control group were non-smoking women, and the three comparator groups were women who quit smoking during the first trimester, women who smoked 1-10 cigarettes per day during pregnancy, and women who smoked more than 10 cigarettes per day during pregnancy. Post-hoc ORs were also calculated within each smoking group, generating ORs for each perinatal outcome in the Aboriginal group, with the non-Aboriginal women as the reference group. Potential interaction effects between SEIFA and cigarette smoking, and asthma and cigarette smoking, were examined. Collinearity between factors was determined with correlation matrices. All analyses were conducted with IBM SPSS version 19 , with $P<0.05$ representing significance.

\section{Results}

Over the 10-year period, 173326 births were recorded to non-Aboriginal women and 4703 were recorded to Aboriginal women in SA. Smoking status during pregnancy was not available in $5580(3 \%)$ of non-Aboriginal and $458(10 \%)$ of Aboriginal pregnancies. Of the remaining 4245 Aboriginal pregnancies, 1655 women (39\%) were non-smokers, 232 (5\%) quit during the first trimester, 1518 (36\%) smoked an average of 1-10 cigarettes per day during pregnancy, and 840 $(20 \%)$ smoked an average of 11 or more cigarettes per day. A greater proportion of the remaining nonAboriginal women were recorded as non-smokers during pregnancy $(n=130026 ; 78 \%$ ), while 9229 women (5\%) quit during the first trimester, 17763 women $(10 \%)$ smoked $1-10$ cigarettes per day in the second trimester, and 10548 women $(6 \%)$ smoked 11 or more cigarettes per day (Appendix $1 ;$ all Appendices online at mja.com. au). Compared with non-Aboriginal women and regardless of smoking status, Aboriginal women had lower maternal age and higher rates of teenage pregnancies, more frequently resided in areas with low SEIFA and were more frequently public hospital patients (Appendix 1). The prevalence of each maternal and neonatal clinical outcome is reported in Appendix 2.

The unadjusted (Appendix 3) and adjusted ORs (Box 1) for each 
2 Adjusted odds ratios $(95 \% \mathrm{Cl})$ for maternal obstetric conditions according to Aboriginal status and smoking during pregnancy, with non-smokers in the matched Aboriginal or non-Aboriginal population as the reference group*

\begin{tabular}{|c|c|c|c|c|c|c|}
\hline \multirow[b]{2}{*}{ Perinatal outcomes } & \multicolumn{2}{|c|}{ Quit smoking } & \multicolumn{2}{|c|}{ Smokes 1-10 cigarettes per day } & \multicolumn{2}{|c|}{ Smokes 11 + cigarettes per day } \\
\hline & Aboriginal & Non-Aboriginal & Aboriginal & Non-Aboriginal & Aboriginal & Non-Aboriginal \\
\hline Hypertensive disorders of pregnancy & $0.88(0.56-1.40)$ & $0.98(0.91-1.06)$ & $0.52(0.40-0.68) \dagger$ & $0.72(0.67-0.77)^{\dagger}$ & $0.63(0.46-0.87) \ddagger$ & $0.70(0.64-0.77)^{\dagger}$ \\
\hline Gestational diabetes & $0.42(0.20-0.89)^{\S}$ & $1.07(0.96-1.20)$ & $0.54(0.39-0.74)^{\dagger}$ & $0.94(0.86-1.03)$ & $0.60(0.42-0.85)^{\ddagger}$ & $1.02(0.92-1.14)$ \\
\hline Antepartum haemorrhage & $1.00(0.47-2.12)$ & $1.01(0.90-1.14)$ & $1.20(0.83-1.72)$ & $1.37(1.27-1.49)^{\dagger}$ & $1.21(0.80-1.83)$ & $1.59(1.44-1.74)^{\dagger}$ \\
\hline Urinary tract infection & $1.47(0.88-2.43)$ & $1.10(0.96-1.25)$ & $1.31(1.00-1.73)$ & $1.30(1.18-1.42)^{\dagger}$ & $1.16(0.83-1.62)$ & $1.42(1.27-1.58)^{\dagger}$ \\
\hline Elective caesarean & $0.86(0.53-1.40)$ & $1.08(1.01-1.16)^{\S}$ & $0.76(0.60-0.96)^{\ddagger}$ & $0.89(0.84-0.93)^{\dagger}$ & $0.67(0.51-0.89)^{\ddagger}$ & $0.88(0.82-0.93)^{\dagger}$ \\
\hline Emergency caesarean & $1.02(0.71-1.47)$ & $1.11(1.05-1.18)^{\dagger}$ & $0.80(0.65-0.97)^{\S}$ & $1.05(1.00-1.10)^{5}$ & $0.93(0.74-1.18)$ & $1.16(1.09-1.24)^{\dagger}$ \\
\hline Premature labour & $1.31(0.74-2.32)$ & $0.96(0.84-1.09)$ & $1.69(1.28-2.23)^{\dagger}$ & $1.46(1.34-1.58)^{\dagger}$ & $1.88(1.37-2.55)^{\dagger}$ & $1.99(1.81-2.18)^{\dagger}$ \\
\hline Premature rupture of membranes & $1.23(0.59-2.54)$ & $1.00(0.85-1.17)$ & $1.31(0.91-1.89)$ & $1.48(1.33-1.64)^{\dagger}$ & $2.13(1.44-3.14)^{\dagger}$ & $1.87(1.65-2.11)^{\dagger}$ \\
\hline
\end{tabular}

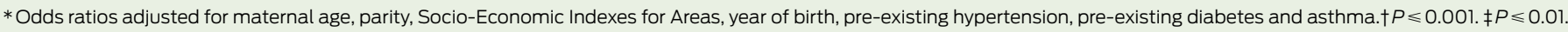
$\oint P \leqslant 0.05$.

perinatal outcome were assessed for Aboriginal women in each smoking group, and non-Aboriginal women with matched smoking status were used as the reference group in each comparison. Regardless of cigarette smoking status, Aboriginal women had an increased risk of urinary tract infections, emergency caesarean sections, premature labour, premature rupture of membranes (PROM), preterm and very preterm birth, IUGR, SGA infants, neonatal intensive care admissions and oxygen therapy, compared with non-Aboriginal women. Among non-smokers, Aboriginal women had an increased risk of all perinatal outcomes compared with non-Aboriginal women, with the exceptions of antepartum haemorrhage and congenital malformations, where the risks were equivalent. Aboriginal women who actively smoked during pregnancy (those who smoked 1-10 or 11 + cigarettes per day) were at increased risk of gestational diabetes, stillbirth and neonatal death compared with non-Aboriginal women with matched smoking status in pregnancy.

The unadjusted (Appendix 4) and adjusted risks (Box 2) of each maternal outcome according to exposure to maternal smoking in pregnancy and Aboriginal status were calculated. Compared with non-smokers, women who actively smoked during pregnancy had a decreased risk of hypertensive disorders of pregnancy and an increased risk of premature labour in both the Aboriginal and non-Aboriginal group, in a dosedependent manner. Active smoking also increased the risk of antepartum haemorrhage, urinary tract infections, emergency caesarean sections and PROM in non-Aboriginal but not Aboriginal women, although smoking $11+$ cigarettes per day increased the risk of PROM in Aboriginal women. Importantly, smoking cessation during the first trimester in both Aboriginal and non-Aboriginal women reversed the risk of antepartum haemorrhage, urinary tract infections, premature labour and PROM to levels comparable with non-smoking women. Regression analyses showed that the interaction between Aboriginal status and smoking was not significant for any maternal outcome except gestational diabetes, where active smoking was associated with a reduction in the adjusted and unadjusted OR in Aboriginal women only (Box 2).

The adjusted (Appendix 5) and unadjusted risks (Box 3 ) of adverse neonatal outcomes were calculated according to exposure to maternal smoking during pregnancy, with non-smoking women as the reference group. Risks for each outcome are presented for Aboriginal and non-Aboriginal women separately. Compared with non-smoking women, active smoking during pregnancy in both Aboriginal and non-Aboriginal women increased the risk of preterm birth, SGA and IUGR in a dose-dependent manner. These risks were reduced when women quit smoking during first trimester to levels equivalent with non-smokers. In non-Aboriginal women, active smoking also increased the risk of very preterm birth, neonatal intensive care, neonatal resuscitation, oxygen therapy and stillbirth, compared with non-smokers. Smoking cessation in the first trimester was associated with no increase in the adjusted risk of any adverse neonatal outcome examined. The interaction of Aboriginal status and smoking was not significant for any neonatal outcome.

\section{Discussion}

Our study examined perinatal outcomes in an Australian population to assess the impact of Aboriginal status and the effect of smoking cessation and active smoking during pregnancy. A higher incidence and risk of poor perinatal outcomes was observed for Aboriginal compared with non-Aboriginal women regardless of smoking status. However, in both populations, active cigarette smoking throughout pregnancy was associated with a further increased risk of adverse maternal and neonatal outcomes compared with those in non-smoking women, including premature labour, PROM, preterm birth, IUGR and SGA infants. Importantly, compared with women who smoked during pregnancy, smoking cessation during the first trimester was associated with a reduction in these risks, clearly demonstrating the benefits of smoking cessation and, to some degree, of reduced smoking in pregnancy for improved maternal and neonatal health outcomes. Despite the higher risk of poor perinatal outcomes in Aboriginal compared with non-Aboriginal pregnancies, cigarette smoking is a modifiable risk factor that significantly contributes to poor outcomes in both populations. 
3 Adjusted odds ratios for neonatal conditions according to Aboriginal status and smoking in pregnancy, with non-smokers in the matched Aboriginal or non-Aboriginal population as the reference group

\begin{tabular}{|c|c|c|c|c|c|c|}
\hline \multirow[b]{2}{*}{ Perinatal outcomes } & \multicolumn{2}{|c|}{ Quit smoking } & \multicolumn{2}{|c|}{ Smokes 1-10 cigarettes per day } & \multicolumn{2}{|c|}{ Smokes 11 + cigarettes per day } \\
\hline & Aboriginal & Non-Aboriginal & Aboriginal & Non-Aboriginal & Aboriginal & Non-Aboriginal \\
\hline Very preterm birth (<32 weeks)* & $1.30(0.65-2.60)$ & $0.89(0.73-1.07)$ & $1.27(0.89-1.80)$ & $1.53(1.37-1.73)^{\dagger}$ & $1.44(0.96-2.16)$ & $1.77(1.54-2.04)^{\dagger}$ \\
\hline Preterm birth (<37 weeks)* & $1.31(0.87-1.99)$ & $0.93(0.85-1.02)$ & $1.40(1.14-1.73)^{\dagger}$ & $1.48(1.39-1.57)^{\dagger}$ & $1.51(1.19-1.93)^{\dagger}$ & $1.87(1.75-2.01)^{\dagger}$ \\
\hline IUGR (<3rd percentile) $)^{\ddagger}$ & $1.33(0.74-2.39)$ & $1.12(0.99-1.26)$ & $2.33(1.77-3.08)^{\dagger}$ & $2.65(2.48-2.83)^{\dagger}$ & $2.81(2.08-3.80)^{\dagger}$ & $3.27(3.03-3.53)^{\dagger}$ \\
\hline SGA (<10th percentile) $)^{\ddagger}$ & $1.28(0.86-1.90)$ & $1.06(0.98-1.14)$ & $2.49(2.06-3.00)^{\dagger}$ & $2.29(2.20-2.40)^{\dagger}$ & $2.50(2.03-3.09)^{\dagger}$ & $2.87(2.73-3.02) \dagger$ \\
\hline Neonatal intensive care ${ }^{\delta}$ & $1.17(0.59-2.34)$ & $0.85(0.72-1.01)$ & $1.19(0.84-1.68)$ & $1.35(1.23-1.52)^{\dagger}$ & $1.27(0.85-1.89)$ & $1.60(1.41-1.82)^{\dagger}$ \\
\hline Neonatal resuscitation ${ }^{\S}$ & $0.94(0.65-1.47)$ & $1.02(0.95-1.10)$ & $0.98(0.79-1.23)$ & $1.16(1.10-1.22)^{\dagger}$ & $0.99(0.75-1.29)$ & $1.26(1.18-1.35)^{\dagger}$ \\
\hline Oxygen therapy (> 4 hours) & $1.03(0.62-1.72)$ & 0.99 (0.90-1.09) & $1.07(0.83-1.37)$ & $1.21(1.13-1.30)^{\dagger}$ & $1.09(0.82-1.47)$ & $1.45(1.34-1.58)^{\dagger}$ \\
\hline Stillbirth* & $0.61(0.14-2.63)$ & $0.97(0.73-1.31)$ & $0.92(0.51-1.65)$ & $1.42(1.17-1.72)^{\dagger}$ & $0.87(0.43-1.76)$ & $1.48(1.16-1.88)^{\dagger}$ \\
\hline Neonatal death (<28 days) & $0.93(0.11-7.56)$ & $0.79(0.47-1.33)$ & $1.19(0.45-3.16)$ & $0.96(0.67-1.36)$ & $1.37(0.44-4.29)$ & $1.22(0.82-1.82)$ \\
\hline Congenital abnormalities* & $1.02(0.42-2.44)$ & $0.89(0.77-1.03)$ & $1.04(0.67-1.61)$ & $1.02(0.92-1.13)$ & $1.40(0.86-2.28)$ & $1.04(0.91-1.19)$ \\
\hline
\end{tabular}

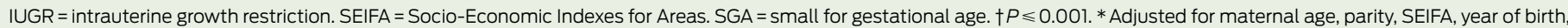

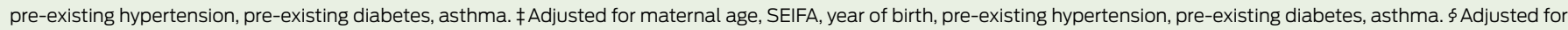

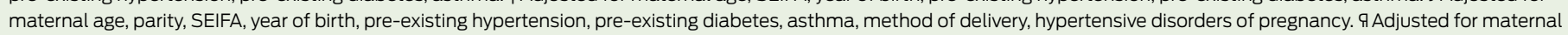
age, parity, SEIFA, year of birth, pre-existing hypertension, pre-existing diabetes, asthma, hypertensive disorders of pregnancy.

This should therefore be prioritised as a component of antenatal care provision in both Aboriginal and nonAboriginal populations to improve perinatal health for mother and child.

It must be acknowledged that the risks of most adverse maternal and neonatal outcomes were greater in Aboriginal than non-Aboriginal pregnancies in all of the smoking strata studied, even after adjusting for potential confounding factors. Other factors clearly contribute to poor outcomes in the Aboriginal population, such as health literacy and inequitable education, remote location and access to health care. Nonetheless, the interaction between smoking and Aboriginal status was not significant for any neonatal or maternal outcome (with the exception of gestational diabetes), indicating that smoking affects Aboriginal and non-Aboriginal pregnancies equally. Smoking during pregnancy did reduce the risk of hypertensive disorders, which is consistent with past research, owing to the effects of smoking on the vasculature, ${ }^{17}$ while the risk reduction for gestational diabetes is likely due to the low maternal weight that frequently occurs with smoking. Nonetheless, it is clear that pregnancy outcomes in all women would improve with smoking cessation in pregnancy.

Our findings support a reduced risk of all adverse neonatal outcomes, as well as most maternal outcomes, in mothers who quit smoking during pregnancy compared with women who continued to smoke. Quit smoking campaigns have achieved lower rates of smoking in the nonAboriginal population; however, they have met with little success in most Aboriginal communities due to a number of social, economic and domestic pressures. ${ }^{8}$ Aboriginal health programs that have successfully reduced pregnancy smoking rates are those operated primarily by Aboriginal health workers. These programs adopt an accessible model of care framework, providing clinical, social, emotional and cultural support. ${ }^{10-14}$ Through such initiatives, the poor perinatal outcomes in our study could be reduced, improving child health.

Two barriers to achieving equitable antenatal care in Aboriginal compared with non-Aboriginal populations are difficulties with access to care ${ }^{18}$ and differences in antenatal service provision. ${ }^{19}$ Compared with nonAboriginal women and women living in urban centres, Aboriginal women attend their first antenatal appointment later in pregnancy and make fewer antenatal visits. ${ }^{20,21}$ Further, they are provided with inconsistent antenatal care services, such as the delivery of routine screening, counselling and provision of health information (including information on smoking cessation). ${ }^{20}$ Our data did not include information relating to the number of antenatal visits attended; however, this measure has since been introduced on the mandatory South Australian birth record form. This will allow future researchers to assess the extent to which this factor contributes to poor perinatal outcomes in Aboriginal women.

Disparities in general health between the Aboriginal and non-Aboriginal Australian populations are recognised. ${ }^{22}$ In our sample, this is evidenced by the increased prevalence of hypertension, diabetes and asthma in Aboriginal compared with non-Aboriginal women. These conditions were included in our regression model when determining adjusted risks, and reduced the unadjusted ORs to some degree. However, the risks associated with cigarette smoking still remained after adjusting for these factors, indicating a strong effect of smoking on perinatal outcomes. Limitations to this analysis include a lack of information about factors that adversely affect pregnancy, including body mass index, diet, chronic renal disease, and alcohol and drug intake before and during pregnancy. While it is important to manage all health conditions in pregnancy, cigarette smoking represents a potential modifiable factor that negatively affects pregnancy maintenance and fetal growth and development. Further, smoking in pregnancy has lifelong health implications for the child, contributing to obesity, metabolic syndrome and reduced lung function. ${ }^{23-25}$ The cessation of cigarette smoking during pregnancy is 
therefore likely to have a profound benefit for both the short-term and long-term health of these children and should be a priority for prepregnancy, antenatal and postnatal health provision for all women. Continued support of health service models that provide such care in an appropriate culturally sensitive setting should be encouraged in order to provide equitable health care.

Acknowledgements: We thank all the midwives, neonatal nurses and other hospital staff who notified to the SA Perinatal Statistics Collection. We acknowledge salary funding support through a National Health and Medical Research Council (NHMRC) Australian Biomedical (Training) Fellowship (1016379) to Nicolette Hodyl and an NHMRC Senior Fellowship (510703) to Vicki Clifton.

Competing interests: No relevant disclosures.

Received 04 Sep 2013, accepted 21 May 2014.

1 Sayers S, Boyle J. Indigenous perinatal and neonatal outcomes: a time for preventive strategies. J Paediatr Child Health 2010; 46: 475-478.

2 Laws P, Sullivan EA. Australia's mothers and babies 2007. Canberra: AlHW, 2009. (AlHW Cat. No. PER 48; Perinatal Statistics Series No. 23.) http://www.aihw.gov.au/publicationdetail/?id=6442468312 (accessed May 2014).

3 Leeds K, Gourley M, Laws P, et al. Indigenous mothers and their babies, Australia 2001-2004. Canberra: Australian Institute of Health and Welfare, 2007. (AIHW Cat No. PER 38; Perinatal Statistics Series No. 19.) http://www.aihw. gov.au/publication-detail/?id=6442468038 (accessed May 2014).

4 Rogers JM. Tobacco and pregnancy. Reprod Toxicol 2009; 28: 152-160.

5 Lumley J, Chamberlain C, Dowswell T, et al. Interventions for promoting smoking cessation during pregnancy. Cochrane Database Syst Rev 2009; (3): CD001055.

6 Briggs VL, Lindorff KJ, Ivers RG. Aboriginal and Torres Strait Islander Australians and tobacco. Tob Control 2003; 12 Suppl 2: ii5-ii8.

7 Johnston V, Thomas DP. What works in Indigenous tobacco control? The perceptions of remote Indigenous community members and health staff. Health Promot J Austr 2010; 21: 45-50.

8 Wood L, France K, Hunt K, et al. Indigenous women and smoking during pregnancy: knowledge, cultural contexts and barriers to cessation. Soc Sci Med 2008; 66: 2378-2389.

9 Passey ME, D’Este CA, Stirling JM, SansonFisher RW. Factors associated with antenata smoking among Aboriginal and Torres Strait Islander women in two jurisdictions. Drug Alcohol Rev 2012; 31: 608-616.

10 Homer CS, Foureur MJ, Allende T, et al. 'It's more than just having a baby' women's experiences of a maternity service for Australian Aboriginal and Torres Strait Islander families. Midwifery 2012; 28: E449-E455.

11 Stamp G, Champion S, Anderson G, et al. Aboriginal maternal and infant care workers: partners in caring for Aboriginal mothers and babies. Rural Remote Health 2008; 8: 883.

12 Panaretto KS, Lee HM, Mitchell MR, et al. Impact of a collaborative shared antenatal care program for urban Indigenous women: a prospective cohort study. Med J Aust 2005; 182 : 514-519.

13 Panaretto KS, Mitchell MR, Anderson L, et al. Sustainable antenatal care services in an urban Indigenous community: the Townsville experience. Med J Aust 2007; 187: 18-22.

14 Adams E, Tongs J. Starting a perinatal and infant mental health service at Winnunga Nimmityjah. Australas Psychiatry 2011; 19 Suppl 1: $S 20-S 22$.

15 McLean A, Scott J, Keane RJ, et al. Validation of the 1994 South Australian Perinatal Data Collection Form. Adelaide: Pregnancy Outcome
Unit, Epidemiology Branch, Department of Human Services, 2001.

16 Australian Bureau of Statistics. Census of Population and Housing: Socio-Economic Indexes for Areas (SEIFA), Australia 2006. Canberra: ABS, 2008. (ABS Cat. No. 2033.0.55.001.)

17 Hodyl NA, Stark MJ, Scheil W, et al. Perinatal outcomes following maternal asthma and cigarette smoking during pregnancy. Eur Respir J 2014; 43: 704-716.

18 Baldwin LM, Grossman DC, Casey S, et al. Perinatal and infant health among rural and urban American Indians/Alaska Natives. Am J Public Health 2002: 92: 1491-1497.

19 Wild K, Maypilama EL, Kildea S, et al. 'Give us the full story': overcoming the challenges to achieving informed choice about fetal anomaly screening in Australian Aboriginal communities. Soc Sci Med 2013; 98: 351-360.

20 Rumbold AR, Bailie RS, Si D, et al. Delivery of maternal health care in Indigenous primary care services: baseline data for an ongoing quality improvement initiative. BMC Pregnancy Childbirth 2011; 11: 16.

21 Roberts CL, Algert CS. The urban and rural divide for women giving birth in NSW, 1990-1997. Aust N Z J Public Health 2000; 24 : 291-297.

22 Australian Bureau of Statistics. The health and welfare of Australia's Aboriginal and Torres Strait Islander peoples, Oct 2010. Canberra: ABS, 2010. (ABS Cat. No. 4704.0.)

23 Oken E, Levitan EB, Gillman MW. Maternal smoking during pregnancy and child overweight: systematic review and metaanalysis. Int J Obes (Lond) 2008; 32: 201-210.

24 Bruin JE, Gerstein HC, Holloway AC. Long-term consequences of fetal and neonatal nicotine exposure: a critical review. Toxicol Sci 2010; 116: 364-374.

25 Skorge TD, Eagan TM, Eide GE, et al. The adult incidence of asthma and respiratory symptoms by passive smoking in uterus or in childhood. Am J Respir Crit Care Med 2005; 172: 61-66. 